\title{
The very steep spectrum radio halo in Abell 697
}

\author{
G. Macario ${ }^{1,2}$, T. Venturi ${ }^{1}$, G. Brunetti ${ }^{1}$, D. Dallacasa ${ }^{1,2}$, S. Giacintucci $^{3,1}$, R. Cassano ${ }^{1}$, S. Bardelli $^{4}$, and R. Athreya ${ }^{5}$ \\ 1 INAF-Istituto di Radioastronomia, via Gobetti 101, 40129 Bologna, Italy \\ e-mail: tventuri@ira.inaf.it \\ 2 Dipartimento di Astronomia, Universitá di Bologna, via Ranzani 1, 40127 Bologna, Italy \\ 3 Harvard-Smithsonian Centre for Astrophysics, 60 Garden Street, Cambridge, MA 02138, USA \\ 4 INAF-Osservatorio Astronomico di Bologna, via Ranzani 1, 40127 Bologna, Italy \\ 5 Indian Institute of Science Education and Research, Sutarwadi Road, Pashan, Pune 411021, INDIA
}

Received 21 January 2010 / Accepted 31 March 2010

\section{ABSTRACT}

\begin{abstract}
Aims. We present a detailed study of the giant radio halo in the galaxy cluster Abell 697 to constrain its origin and connection with the cluster dynamics.

Methods. We performed high sensitivity GMRT observations at $325 \mathrm{MHz}$, which showed that the radio halo is much brighter and larger at this frequency than in previous $610 \mathrm{MHz}$ observations. To derive the integrated spectrum in the frequency range $325 \mathrm{MHz}-1.4 \mathrm{GHz}$, we reanalysed archival VLA data at $1.4 \mathrm{GHz}$ and used proprietary GMRT data at $610 \mathrm{MHz}$.

Results. Our multifrequency analysis shows that the total radio spectrum of the giant radio halo in A697 is very steep, with $\alpha_{325 \mathrm{GHz}}^{1.4 \mathrm{GHz}} \approx 1.7-1.8$. Owing to energy arguments, a hadronic origin of the halo is disfavoured by this steep spectrum. Very steep spectrum halos in merging clusters are predicted in the case that the emitting electrons are accelerated by turbulence. Observations with upcoming low frequency arrays will be able to test these expectations.
\end{abstract}

Key words. radiation mechanism: non-thermal - galaxies: clusters: general - galaxies: clusters: individual: Abell 697

\section{Introduction}

Radio halos are diffuse low surface brightness radio sources extended on Mpc scales, observed in the central regions of a fraction of X-ray luminous (i.e., massive) galaxy clusters. Their synchrotron emission is not associated with individual galaxies, but originates in the non-thermal component of the intracluster medium (ICM). They provide the most important piece of evidence for relativistic $(\sim \mathrm{GeV})$ electrons and $\mu \mathrm{G}$ magnetic fields in galaxy clusters (see Ferrari et al. 2008 for a review). To date radio halos have been found from large sky surveys (such as the Northern VLA Sky Survey, NVSS, Condon et al. 1998 and the Westerbork Northern Sky Survey, WENSS, Rengelink et al. 1997; i.e., Giovannini et al. 1999; Kempner \& Sarazin 2001), and from pointed observations of individual clusters, mostly carried out at 1.4 GHz (Govoni et al. 2001; Bacchi et al. 2003; and the compilation of Giovannini et al. 2009) and $610 \mathrm{MHz}$ (the GMRT Radio Halo survey, Venturi et al. 2007 and 2008, hereinafter VGB07 and VGD08). They have steep radio spectra, with $\alpha \sim 1.2-1.4\left(S \propto v^{-\alpha}\right)$ from integrated flux density measurements.

The origin of radio halos has long been unknown, since the radiative lifetime of the relativistic electrons responsible for their synchrotron emission is much shorter than the particle diffusion time required to cover their Mpc extent (Jaffe 1977). This requires some form of particle re-acceleration. Two main models have been proposed to explain the origin of radio halos: (1) the "re-acceleration model", whereby radio halos originate from the in-situ re-acceleration of pre-existing electrons by magneto-hydrodynamic (MHD) turbulence injected into the ICM during cluster mergers (Brunetti et al. 2001; Petrosian 2001; Brunetti \& Blasi 2005); (2) "secondary electron models", predicting that relativistic electrons are injected into the cluster volume by hadronic collisions between relativistic cosmic rays and the thermal protons of the ICM (e.g. Dennison 1980; Blasi \& Colafrancesco 1999).

Radio observations provide indirect evidence of turbulent acceleration in the ICM (e.g. Brunetti et al. 2008). Observational support for the "re-acceleration scenario" is also provided by the growing evidence that cluster mergers and radio halos are related, based on high sensitivity radio and X-ray observations (Buote 2001; Govoni et al. 2004; VGD08 and references therein; Giacintucci et al. 2009; Cassano 2009), and by constraints on the evolution of radio halos (Brunetti et al. 2009).

The analysis of the radio spectrum of halos is important to investigating their origin. The turbulent re-acceleration model provides the unique expectation of spectra much steeper than those found to date, as a consequence of less energetic merger events (e.g. Cassano 2009). The detection of radio halos with very steep spectrum $(\alpha>1.6)$ would be a major piece of evidence supporting this scenario, and at the same time would disfavour a secondary origin of the electrons, which requires a very large proton energy budget (e.g. Brunetti 2004). The prototype of these sources, which we refer to as ultra steep spectrum radio halos (hereinafter USSRH), has been recently discovered in the merging cluster A 521 and has a spectrum with $\alpha \sim 1.9$ (Brunetti et al. 2008; Dallacasa et al. 2009).

In this paper, we present a detailed study of the merging cluster A 697, which hosts a candidate steep spectrum radio halo. Our work is based on proprietary GMRT radio observations at 610 and $325 \mathrm{MHz}$, and data from the VLA public archive at $1.4 \mathrm{GHz}$.

A 697 is a rich and massive galaxy cluster located at $z=$ 0.282 , belonging to the Abell, Corwin, and Olowin catalogue 
Table 1. General properties of the galaxy cluster A 697.

\begin{tabular}{ll}
\hline \hline $\mathrm{RA}_{J 2000}$ & $08 \mathrm{~h} 42 \mathrm{~m} 53.3 \mathrm{~s}$ \\
$\operatorname{Dec}_{J 2000}$ & $+36^{\circ} 20^{\prime} 12^{\prime \prime}$ \\
Bautz-Morgan Class & II-III \\
Richness & 1 \\
$z$ & 0.282 \\
$\sigma_{v}$ & $1334 \mathrm{~km} \mathrm{~s}^{-1 a}$ \\
$L_{\mathrm{X}[0.1-2.4 \mathrm{keV}]}$ & $10.57 \times 10^{44} \mathrm{erg} \mathrm{s}^{-1}$ \\
$M_{\mathrm{V}}$ & $2.25 \times 10^{15} M_{\odot}^{b}$ \\
$R_{\mathrm{V}}$ & $2.90 \mathrm{Mpc}^{b}$ \\
\hline
\end{tabular}

Notes. ${ }^{(a)}$ Girardi et al. (2006); ${ }^{(b)}$ Estimated from the $L_{\mathrm{X}}-M_{\mathrm{V}}$ relation, see Eqs. (6) and (7) in Cassano et al. (2006).

(ACO catalogue, Abell et al. 1989). The cluster is hot and luminous in the X-ray band, and is part of the ROSAT Brightest Cluster Sample (BCS; Ebeling et al. 1998). Its general properties are summarized in Table $1^{1}$, which provides coordinates, morphological classification of the cluster and richness, redshift $z$, cluster velocity dispersion $\sigma_{v}$ (from optical spectroscopy), X-ray luminosity $L_{\mathrm{X}}$ (taken from the BCS catalogue), virial mass $M_{\mathrm{V}}$, and the corresponding virial radius $R_{\mathrm{V}}$. Diffuse cluster-scale radio emission in A 697 was first suggested by Kempner \& Sarazin (2001) after inspection of the NVSS and the WENSS, and confirmed by observations at $610 \mathrm{MHz}$ with the Giant Metrewave Radio Telescope (GMRT) as part of the GMRT Radio Halo Survey (VGB07 and VGD08). From those observations, the extended radio emission at the cluster centre was unambiguously classified as a giant ${ }^{2}$ radio halo.

The paper is organized as follows. In Sect. 2, we present the new $325 \mathrm{MHz}$ GMRT data; in Sects. 3 and 4, we derive the integrated spectrum and discuss the sources of uncertainty in the flux density measurements; in Sect. 5, we discuss the origin of the A 697 radio halo in the framework of the turbulent re-acceleration model. Summary and conclusions are given in Sect. 6.

\section{GMRT radio data at $325 \mathrm{MHz}$}

\subsection{Radio observations and data reduction}

The main characteristics of the $325 \mathrm{MHz}$ observations are summarised in Table 2, which reports observing date, frequency, total bandwidth, total time on source, synthesized half power beamwidth (HPBW), rms level $(1 \sigma)$ at full resolution, and $u-v$ range of the full dataset.

The observations were carried out using simultaneously the upper and lower side bands (USB and LSB, respectively), for a total observing bandwidth of $32 \mathrm{MHz}$. The default spectralline observing mode was performed, with 128 channels for each band and a spectral resolution of $125 \mathrm{kHz} / \mathrm{channel}$. The USB and LSB datasets were calibrated and analysed separately using the NRAO Astronomical Image Processing System package (AIPS). We used 3C 147 and 3C 286 as primary calibrators, which were observed respectively at the beginning and the end of the observing run. The point source $0735+331$ was used as a phase calibrator.

\footnotetext{
1 We adopt the $\Lambda$ CDM cosmology with $H_{0}=70 \mathrm{~km} \mathrm{~s}^{-1} \mathrm{Mpc}^{-1}, \Omega_{\mathrm{m}}=$ 0.3 , and $\Omega_{\Lambda}=0.7$. At the redshift of A $697(z=0.282)$, this cosmology leads to a linear scale of $1^{\prime \prime}=4.26 \mathrm{kpc}$.

${ }^{2}$ Linear size $\gtrsim 1 \mathrm{Mpc} \mathrm{h}_{50}^{-1}$.
}

Owing to the considerably lower quality of the LSB data (strong RFI residuals), only the USB data were used to produce the final images presented and analysed in this paper.

The very large field of view of the GMRT at $325 \mathrm{MHz}$ (primary beam $\sim 1.8^{\circ}$ ) required the implementation of the wide-field imaging technique in each step of the self-calibration process, to account for the non-planar nature of the sky. We covered a field of view as large as $\sim 2.7 \times 2.7$ square degrees with 25 facets, to include possible strong sources located beyond the primary lobe, conventionally cut at 5\%. We initially self-calibrated the longest baselines using only the point sources in the field, then we progressively included short baselines and resolved radio sources. We finally included also the emission from the radio halo. We note that only phase self-calibration was applied.

Even though only half of the full dataset was usable, the quality of the final image is very good: the rms ranges from $\sim 45 \mu \mathrm{Jy} \mathrm{b}^{-1}$ to $\sim 55 \mu \mathrm{Jy} \mathrm{b}^{-1}$. Despite the relatively low frequency, confusion in this image is negligible, due to the arcsecond resolution.

The residual amplitude errors for each individual antenna are $\$ 5 \%$. On the basis of this result, we can conservatively assume that the absolute flux density calibration is within $5 \%$. This value also accounts for the uncertainty in the calibrator flux density scale.

\subsection{The field}

In Fig. 1, we report the $325 \mathrm{MHz}$ GMRT full resolution contours of the central $12^{\prime} \times 12^{\prime}\left(\sim 3 \times 3 \mathrm{Mpc}^{2}\right)$ portion of the A 697 field. The region corresponds to half the cluster virial radius $\left(R_{\mathrm{V}}=\right.$ $2.9 \mathrm{Mpc}$, Table 1). The most prominent features at this resolution are the two extended radio galaxies, labelled as $\mathrm{S} 1$ and $\mathrm{S} 2$ in Fig. 1, located south of the cluster centre. In addition, the diffuse radio emission associated with the radio halo is clearly visible around the cluster centre.

\subsection{The radio halo}

Figure 2 zooms into the central cluster region. The left panel shows the full resolution $325 \mathrm{MHz}$ contours (starting from $\pm 3 \sigma$ ), overlaid on the red optical frame from the Digitized Palomar Sky Survey (DSS-2). In addition to the individual radio sources with optical counterparts, diffuse emission is clearly visible at the cluster centre. Three discrete radio sources embedded in the halo emission are optically identified with cluster members (Girardi et al. 2006, hereinafter G06). We labelled them A, D, and $\mathrm{G}$, following the same notation used for the corresponding sources identified in the GMRT $610 \mathrm{MHz}$ full resolution image (left panel of Fig. 2, see also VGD08). In Table 3, we report their radio position and flux density at $325 \mathrm{MHz}$ (obtained from Gaussian fits to the full resolution image), and their optical identification and redshift (from G06). Source A is identified with the central cD galaxy.

To properly image the diffuse emission of the radio halo, the three sources A, D, and G, whose total flux density is $S_{325 \mathrm{MHz}}=$ $7.96 \mathrm{mJy}$, were subtracted from the $u-v$ data. The final "subtracted" dataset was used to produce images of the radio halo at various angular resolutions, tapering the $u-v$ data by means of the parameters robust and uvtaper in the task IMAGR.

In the right panel of Fig. 2, we report the $325 \mathrm{MHz}$ contours of the radio halo at the resolution of $46.8^{\prime \prime} \times 41.4^{\prime \prime}$, overlaid on a GMRT $610 \mathrm{MHz}$ image of similar resolution and obtained with a comparable weighting scheme $\left(46.4^{\prime \prime} \times 35.9^{\prime \prime}\right.$; grey scale, see 
G. Macario et al.: The very steep spectrum radio halo in Abell 697

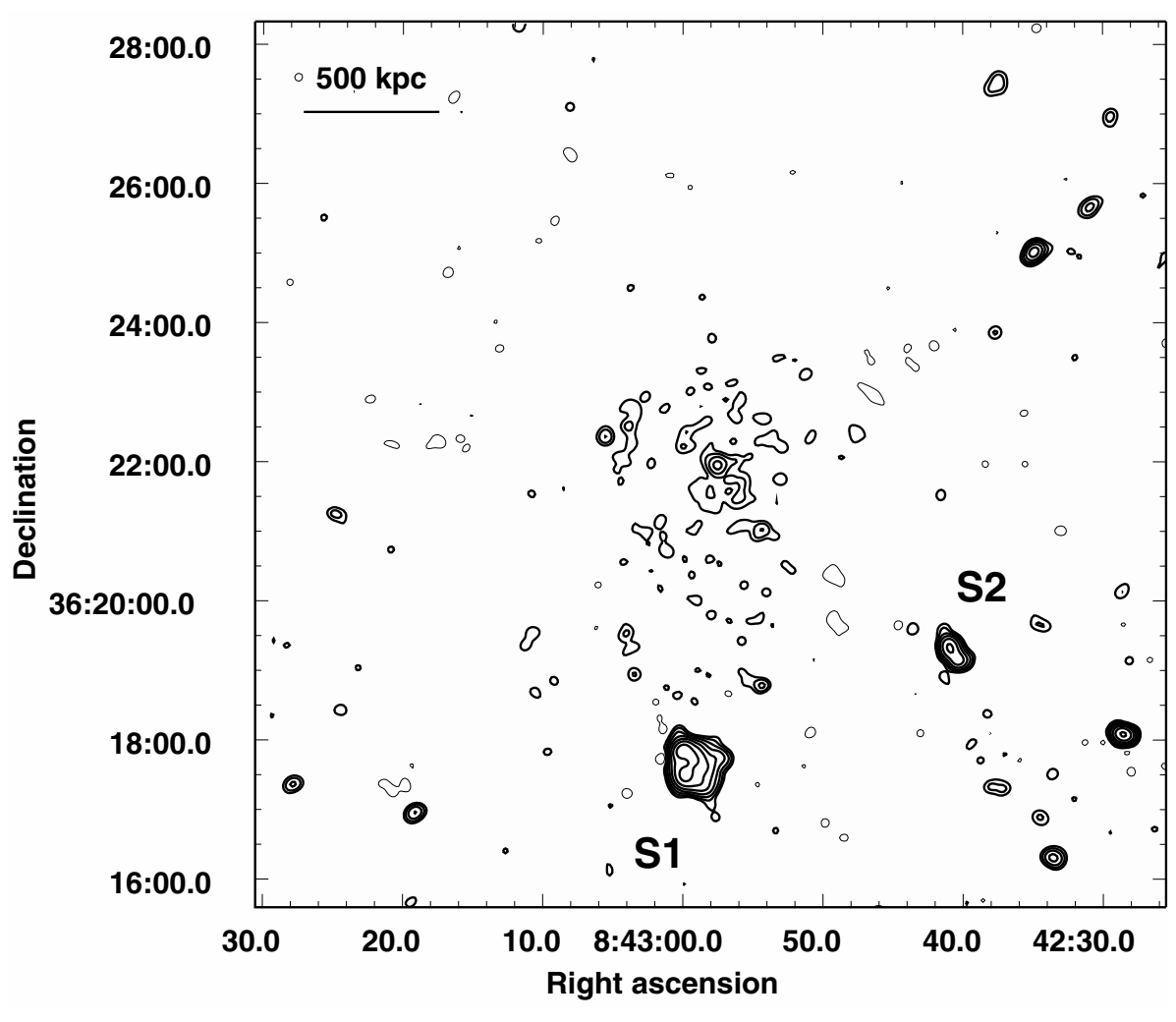

Fig. 1. GMRT $325 \mathrm{MHz}$ radio contours of the inner $12^{\prime} \times 12^{\prime}$ region centered on A 697 . The $1 \sigma$ level in the image is $45 \mu \mathrm{Jy} \mathrm{b}^{-1}$. Contours are spaced by a factor 2 starting from $5 \sigma=$ $\pm 0.225 \mathrm{mJy} \mathrm{b}^{-1}$. The HPWB is $10.0^{\prime \prime} \times 9.1^{\prime \prime}$, position angle (PA) $-64^{\circ}$. The labels $\mathrm{S} 1$ and $\mathrm{S} 2$ indicate the two extended radio galaxies in the southern part of the cluster.

Table 2. Summary of the GMRT radio observations.

\begin{tabular}{cclcccc}
\hline \hline $\begin{array}{c}\text { Observation } \\
\text { date }\end{array}$ & $\begin{array}{c}v \\
(\mathrm{MHz})\end{array}$ & $\begin{array}{l}\Delta v \\
(\mathrm{MHz})\end{array}$ & $\begin{array}{c}t \\
(\mathrm{~min})\end{array}$ & $\begin{array}{c}\text { HPBW, PA } \\
\left(\text { full array },{ }^{\prime \prime} \times^{\prime \prime},{ }^{\circ}\right)\end{array}$ & $\begin{array}{c}\text { rms } \\
\left(\mu \mathrm{Jy} \mathrm{b}{ }^{-1}\right)\end{array}$ & $\begin{array}{c}u-v \text { range } \\
(\mathrm{k} \lambda)\end{array}$ \\
\hline 17 Jan. 2007 & 325 & $32(16)^{*}$ & 480 & $10.0 \times 9.1,-64$ & 45 & $\sim 0.08-25$ \\
\hline
\end{tabular}

Notes. ${ }^{(*)}$ The observations were performed using a total bandwidth of $32 \mathrm{MHz}$ (USB+LSB), but only the USB dataset was used for the analysis (see Sect. 2.1).

also VGD08). The first contour corresponds to the $\pm 3 \sigma$ significance level.

The radio halo of A 697 is very extended at $325 \mathrm{MHz}$, its largest angular size (LAS) being $\sim 5.1^{\prime}$, corresponding to a largest linear size (LLS) of $\sim 1.3 \mathrm{Mpc}_{70}^{-1}$. Its overall morphology is regular and symmetric, and more extended than when imaged at $610 \mathrm{MHz}$. The central $\sim 1^{\prime}$ is similar at both frequencies, and the bright feature clearly visible at $610 \mathrm{MHz}$ in the western part of the halo is almost coincident with a similar structure at $325 \mathrm{MHz}$.

The total integration time of the $610 \mathrm{MHz}$ observations is much shorter than that at $325 \mathrm{MHz}$ (see Sect. 2.1), and the $u-v$ coverage of the short spacings is poorer. In Fig. 3, we compare the inner portions of the $u-v$ plane at 325 and $610 \mathrm{MHz}$ (left and right panel, respectively); visibilities relative to baselines shorter than $1 \mathrm{k} \lambda$ are shown ${ }^{3}$. For this reason, a detailed spectral index imaging was not carried out.

The total flux density of the radio halo at $325 \mathrm{MHz}$ is $S_{325 \mathrm{MHz}}=47.3 \mathrm{mJy}$ (see Sect. 4 for a detailed discussion of the uncertainties associated with this value); it was obtained by integrating the low resolution image (Fig. 2, right panel) over the region covered by the $\sim 3 \sigma$ contour. The corresponding total radio power is $\log P_{325 \mathrm{MHz}}(\mathrm{W} / \mathrm{Hz})=25.07$.

3 The angular size of the halo $\left(\sim 5^{\prime}\right)$ is sampled by visibilities corresponding to baselines shorter than $\sim 0.7 \mathrm{k} \lambda$.

\section{The integrated radio spectrum of the halo}

To derive the spectrum of the giant radio halo in A 697 with at least three data points, we complemented the GMRT flux density values at $610 \mathrm{MHz}$ and $325 \mathrm{MHz}$ with archival $1.4 \mathrm{GHz}$ VLA-C public data.

\subsection{VLA archive data at $1.4 \mathrm{GHz}$}

We reanalysed archival VLA-C observations at $1.4 \mathrm{GHz}$ (Obs. Id. AJ0252). These are short observations centered on A 697, of total integration time $\sim 50 \mathrm{~min}$ and $u-v$ range $0.3-15 \mathrm{k} \lambda$. After the usual a-priori calibration, the final images were obtaind after a few iterations of phase-only self-calibration.

We successfully detected diffuse emission at the cluster centre. To account for the contribution of the point sources in the halo region, we produced an image using only baselines longer than $3 \mathrm{k} \lambda$, to exclude the contribution of the halo emission (resolution $\left.\sim 9^{\prime \prime} \times 11^{\prime \prime}\right)$. The $1 \sigma \mathrm{rms}$ is $\sim 12 \mu \mathrm{Jy} \mathrm{b}^{-1}$. In the central halo region, we identified 7 point sources, with peak flux density exceeding 5 times the rms level; two of them had already been found at $610 \mathrm{MHz}$, and labelled A (the central cD) and F in VGD08. We fitted all sources with individual Gaussians, and obtained a total contribution to the integrated flux density of $S_{1.4 \mathrm{GHz}}=0.97 \mathrm{mJy}$. In Fig. 4, we show a low resolution image of the radio halo at $1.4 \mathrm{GHz}$ (restoring beam of $35^{\prime \prime} \times 35^{\prime \prime}$ ) 

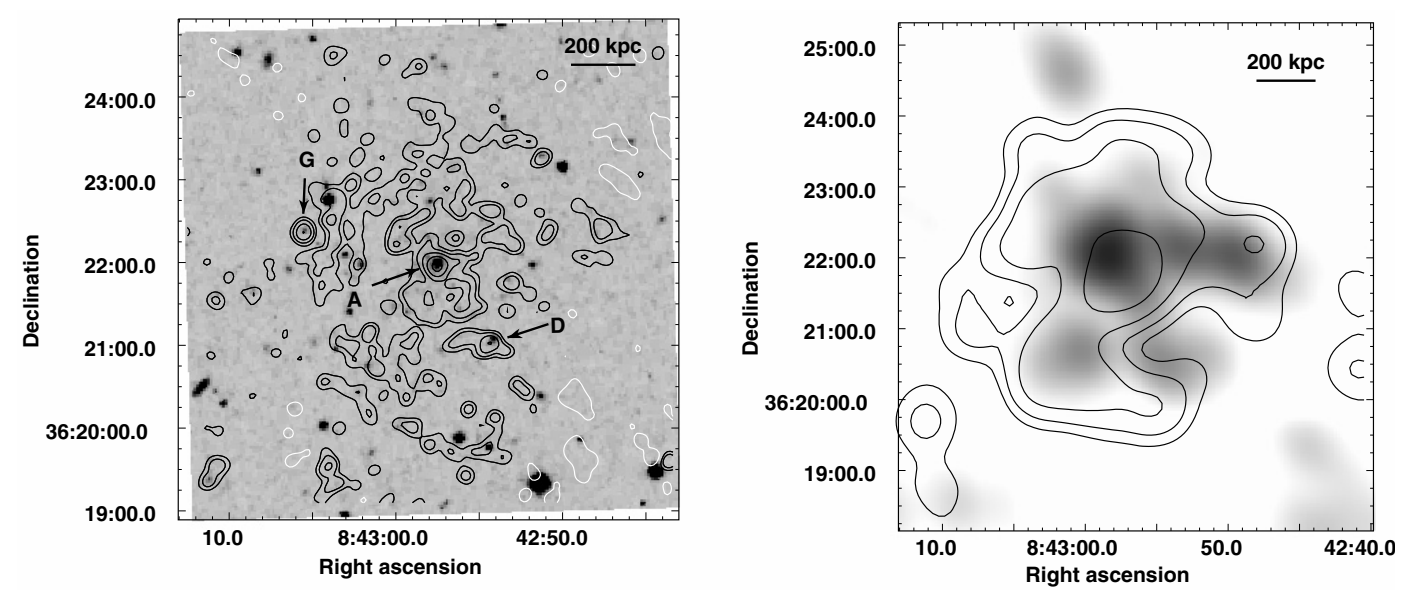

Fig. 2. Left - Full resolution GMRT $325 \mathrm{MHz}$ contours of the central region of A 697, superimposed on the Second Palomar Sky Survey (POSS-2) optical image. The image is the same as in Fig. $1\left(10.0^{\prime \prime} \times 9.1^{\prime \prime}, \mathrm{PA}-64^{\circ}, 1 \sigma\right.$ level is $\left.0.045 \mathrm{mJy} \mathrm{b}^{-1}\right)$ and contours are spaced by a factor of 2 , starting from $\pm 0.135 \mathrm{mJy} \mathrm{b}^{-1}(3 \sigma)$. Individual radio sources are labelled by letters A, D, and G (as in VGD08, Fig. 2). Right - Low resolution GMRT image at $325 \mathrm{MHz}$ of the radio halo (obtained after subtraction of the individual sources, see Sect. 2.3) overlaid on the GMRT 610 MHz image (grey scale). The resolution of the $325 \mathrm{MHz}$ image is $46.8^{\prime \prime} \times 41.4^{\prime \prime}, \mathrm{PA} 80^{\circ}$, and the $1 \sigma$ noise level is $0.15 \mathrm{mJy} \mathrm{b}^{-1}$. Logarithmic contours are shown, starting from $\pm 0.45 \mathrm{mJy} \mathrm{b}^{-1}$. The $610 \mathrm{MHz}$ image has a resolution of $46.4^{\prime \prime} \times 35.9^{\prime \prime}, \mathrm{PA} 42^{\circ}$, and the $1 \sigma$ noise level is $0.05 \mathrm{mJy} \mathrm{b}$.
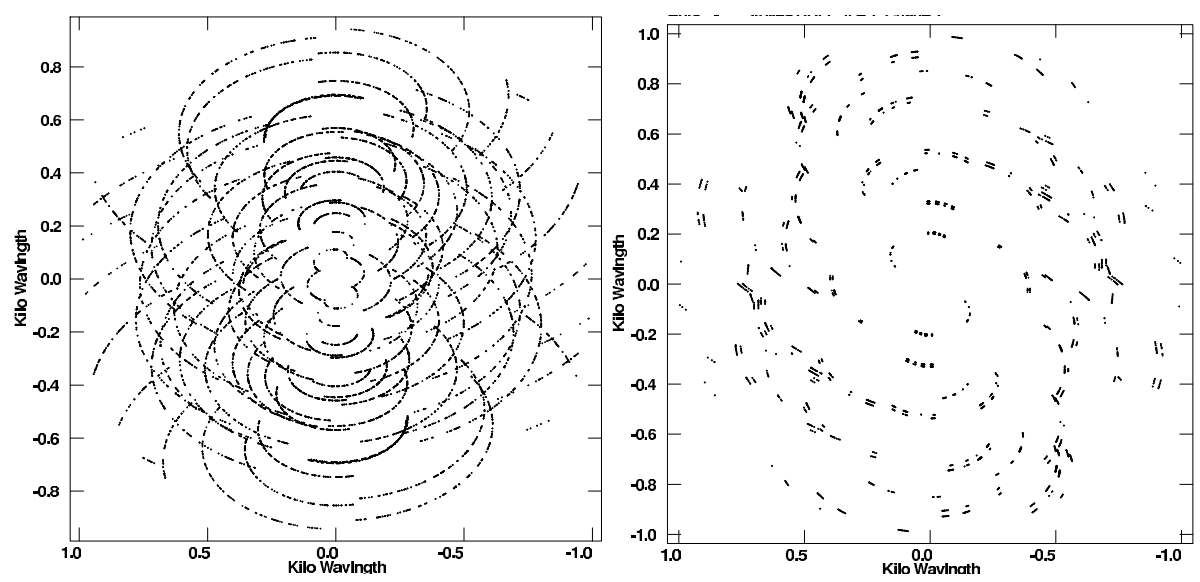

Fig. 3. Comparison between the inner portion of the $u-v$ plane sampled by the GMRT observations at $325 \mathrm{MHz}$ (left panel) and at $610 \mathrm{MHz}$ (right) from which the flux density measurements of the halo have been taken. Only baselines shorter than $1 \mathrm{k} \lambda$ have been plotted.

Table 3. Individual radio galaxies at the center of A 697.

\begin{tabular}{lcccc}
\hline \hline $\begin{array}{l}\text { Radio } \\
\text { source }\end{array}$ & $\begin{array}{c}\text { Radio position } \\
\mathrm{RA}_{J 2000} \& \mathrm{Dec}_{J 2000}\end{array}$ & $\begin{array}{c}S_{325 \mathrm{MHz}} \\
\text { mJy }\end{array}$ & $\begin{array}{c}\text { Optical position } \\
\mathrm{RA}_{J 2000} \& \mathrm{Dec}_{J 2000}\end{array}$ & $z$ \\
\hline $\mathrm{A}$ & $084257.70+362201$ & $4.63 \pm 0.23$ & $084257.55+362200$ & 0.281 \\
$\mathrm{D}$ & $084254.54+362105$ & $1.97 \pm 0.10$ & $084254.36+362103$ & 0.274 \\
$\mathrm{G}$ & $084305.65+362225$ & $1.36 \pm 0.07$ & $084305.50+362224$ & 0.267 \\
\hline
\end{tabular}

for comparison with the $325 \mathrm{MHz}$ and $610 \mathrm{MHz}$ images. The crosses mark the position of the embedded radio point sources. We note that the field shown is the same as the right panel of Fig. 2.

At $1.4 \mathrm{GHz}$, the radio halo appears considerably smaller than in the GMRT images. It is elongated in the east-west direction, in agreement with the brightest part of the $610 \mathrm{MHz}$ emission. The LAS is $\simeq 190^{\prime \prime}$, corresponding to a LLS $\simeq 810 \mathrm{kpc} \mathrm{h}_{70}^{-1}$. Its total flux density is $S_{1.4 \mathrm{GHz}}=3.7 \mathrm{mJy}$, corresponding to a radio power $\log P_{1.4 \mathrm{GHz}}(\mathrm{W} / \mathrm{Hz})=23.95$. This value was obtained by integrating this image over the same area covered by the radio halo at $325 \mathrm{MHz}$, and after subtraction of the total contribution of the embedded point sources.

Hints of diffuse emission at the centre of A 697 are visible in the NVSS $1.4 \mathrm{GHz}$ image (Fig. 5, left panel). However, after an accurate inspection we concluded that this image is affected by fringe residuals (see Giacintucci 2007). For this reason, the NVSS pointing containing the A 697 field was imaged after a new calibration. We found out that one IF was affected by strong interference, and it was necessary to remove it from the selfcalibration and imaging process. The new image is reported in the central panel of Fig. 5: no extended emission is indeed detected at the cluster centre at the noise level of $\sim 0.3 \mathrm{mJy} \mathrm{b}^{-1}$. Our reanalysis confirmed that the extended structure visible in the public NVSS image is coincident with a peak of a residual fringe, which crosses the image along the NW-SE direction, i.e., the same direction as that of the structure itself. Some residual patterns are still visible in the recalibrated image at the $1 \sigma$ level. The right panel of Fig. 5 reports the recalibrated NVSS image (contours) overlaid on the $1.4 \mathrm{GHz}$ VLA-C image (grey scale). The non-detection on the recalibrated NVSS image is consistent with the VLA-C image, given the rms, peak, and restoring 
G. Macario et al.: The very steep spectrum radio halo in Abell 697

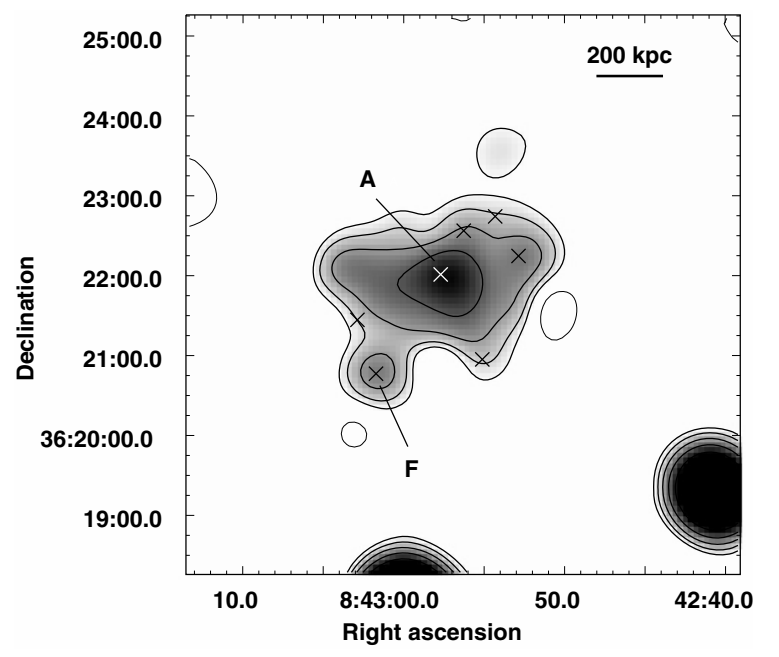

Fig. 4. Low resolution VLA-C $1.4 \mathrm{GHz}$ image of the radio halo in A 697. The $1 \sigma$ level in the image is $25 \mu \mathrm{Jy} \mathrm{b}^{-1}$. Contours are spaced by a factor 2 starting from $3 \sigma= \pm 0.075 \mathrm{mJy} \mathrm{b}^{-1}$. The restoring beam is $35.0^{\prime \prime} \times 35.0^{\prime \prime}, \mathrm{PA} 0^{\circ}$. Crosses mark the position of the radio sources embedded in the halo emission.

beams in the two images (see figure caption). We integrated the flux density on the recalibrated NVSS image over the same sky portion covered by the radio halo at $1.4 \mathrm{GHz}$, and measured $2.4 \mathrm{mJy}$. Considering that the contribution of point sources in this region is $0.97 \mathrm{mJy}$ (see above), we can conclude that the flux density of the radio halo on the NVSS is equal to $\simeq 1.4 \mathrm{mJy}$.

\subsection{Integrated radio spectrum}

The observed integrated spectrum of radio halos may be affected by two main factors: (1) the contribution to the flux density of embedded individual radio sources, and (2) the $u-v$ coverage at short spacings. Embedded individual sources are probably of AGN origin, whose spectrum is flatter than radio halos. An inaccurate or inefficient subtraction of these sources may introduce a scatter of the flux density measurements at the various frequencies. On the other hand, the $u-v$ coverage at short spacings may affect the detection of large-scale structure.

We dealt with factor (1) in Sects. 2.3 and 3.1. Factor (2) is the focus of Sect. 4.

All the available flux density measurements of the radio halo are reported in Table 4, along with the angular resolution of the images used for the measurements. The source is undetected in the Very Low-Frequency Sky Survey (VLSS), most likely due to the very poor sensitivity of the A 697 field.

The sources of uncertainty in the radio halo flux densities at $325 \mathrm{MHz}$ and $1.4 \mathrm{GHz}$ are the calibration errors and the procedure of point source subtraction, which we estimate equal $\sim 5 \%$. The flux density value at $610 \mathrm{MHz}$ is affected by a higher uncertainty. We performed an accurate check on flux density at $325 \mathrm{MHz}, 610 \mathrm{MHz}$, and $1.4 \mathrm{GHz}$ for a number of discrete sources in the central field (see Fig. 1), using three images with the same angular resolution as the full resolution at $1.4 \mathrm{GHz}$ $\left(\sim 16^{\prime \prime} \times 16^{\prime \prime}\right)$, after correction for the corresponding primary beam. We found that the $610 \mathrm{MHz}$ measurements are systematically underestimated by $\sim 12 \%$; after inspection of the data, we concluded that this is caused by a sistematic error in the amplitude. We note that large residual calibration errors at $610 \mathrm{MHz}$ were also reported for the cluster A 3562 in Giacintucci et al. (2005). Taking all this into consideration, the $610 \mathrm{MHz}$ flux
Table 4. Flux densites of the radio halo in A 697.

\begin{tabular}{cccc}
\hline \hline $\begin{array}{c}v \\
(\mathrm{MHz})\end{array}$ & $\begin{array}{c}S_{v} \\
(\mathrm{mJy})\end{array}$ & $\begin{array}{c}\text { HPBW } \\
" \times "\end{array}$ & Ref. \\
\hline 325 & $47.3 \pm 2.7$ & $46.8 \times 41.4$ & This work; Fig. 2 \\
610 & $14.6 \pm 1.7$ & $46.4 \times 35.9$ & VGD08; see also this work Fig. 2 \\
1400 & $3.7 \pm 0.3$ & $35.0 \times 35.0$ & This work; Fig. 4* \\
\hline
\end{tabular}

Notes. ${ }^{(*)}$ From VLA-C archival data (Obs. Id. AJ0252).

density value of the radio halo in A 697 given in VGD08 was corrected to account for this error (Table 4 reports the new corrected value).

The integrated radio spectrum of the halo is shown in Fig. 6. The red solid line is the linear fit to the data (filled triangles) weighted for the uncertainties. A single power-law fit to the data provides a spectral index $\alpha=1.8 \pm 0.1^{4}$.

\section{Uncertainties in the spectral slope}

The combination of very low brightness emission and large angular size makes the imaging of faint radio halos a challenging process. The sampling of the $u-v$ coverage at short spacings is essential for a reliable imaging of extended and diffuse radio sources, and this is particularly relevant for faint radio halos, as is the case of A 697. The most serious effect of an inadequate $u-v$ coverage at short baselines is the loss of a fraction of the radio halo flux density, which may affect not only the imaging at an individual frequency, but also the integrated spectral index of the source. This effect becomes even more severe when only a few data points over a small frequency range are available.

We discuss the role of the $u-v$ coverage in the GMRT observations of A 697 and the implications on the spectral steepness of its giant radio halo. We show that part of the total flux density of the radio halo could not be accounted for by the GMRT observations. This effect is more severe at $610 \mathrm{MHz}$, since Fig. 3 clearly shows that the inner portion of the $u-v$ coverage is much more finely sampled in the $325 \mathrm{MHz}$ observations. Given that an underestimate of the $610 \mathrm{MHz}$ flux density would produce a steeper spectrum, it is crucial that we test the reliability of the spectral index derived in the present work.

To constrain the spectral steepness of the radio halo, we estimated the flux density losses expected in both the 325 and $610 \mathrm{MHz}$ GMRT data following the "fake" radio halo procedure, described in detail in VGD08. A "fake radio halo" is a model of the radio halo brightness profile, which consists of a set of optically thin concentric spheres of different radius and flux density. Here we briefly describe the main steps of this procedure and the results obtained.

\subsection{The $325 \mathrm{MHz}$ data}

To evaluate the flux density loss in the halo at $325 \mathrm{MHz}$, families of fake radio halos were injected into the $u-v$ data. We chose different sets of values for the total flux density and largest angular scale, and injected the fake halos into a region of the field free of point sources, and close enough to the cluster centre to

\footnotetext{
${ }^{4}$ Giovannini et al. (2009) estimated a spectral index $\alpha_{325 \mathrm{MHz}}^{1.4 \mathrm{GHz}}=1.2$ using our GMRT $325 \mathrm{MHz}$ flux density value preliminary presented in Venturi et al. (2009) and the $1.4 \mathrm{GHz}$ value derived from the original NVSS image. However, as shown in Sect. 3.1, the original NVSS value overestimates the flux density at the centre of A 697.
} 

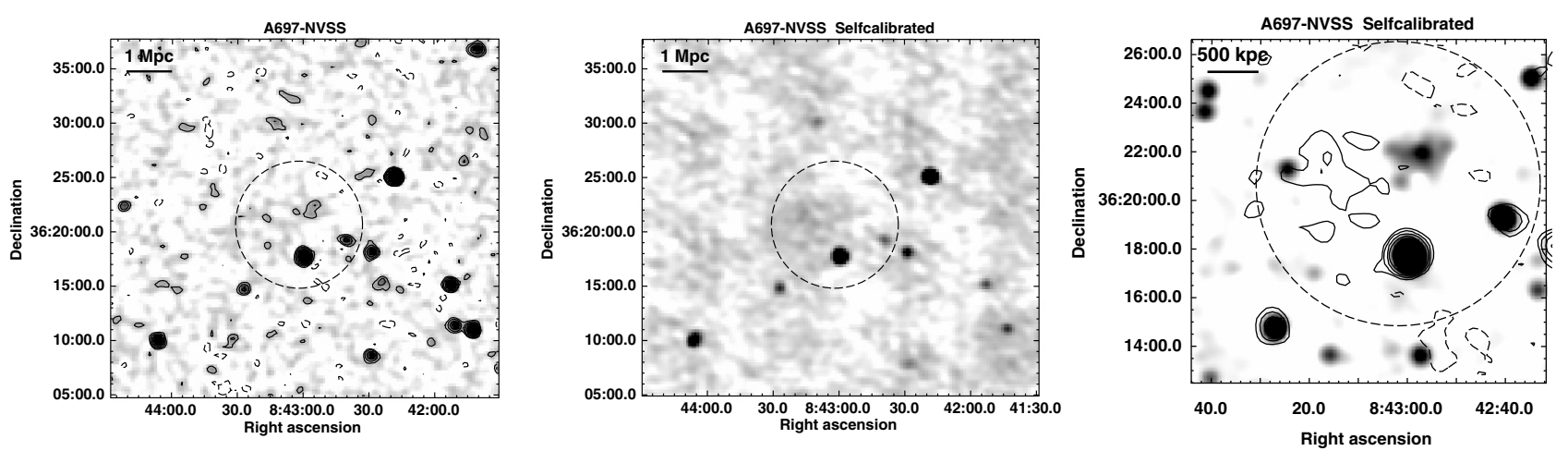

Fig. 5. Left panel: radio contours of the A 697 field from the public $1.4 \mathrm{GHz}$ NVSS image (grey scale). The $1 \sigma$ level is $0.45 \mathrm{mJy} \mathrm{b} \mathrm{b}^{-1}$. Contour levels are spaced in intervals of a factor of 2 , starting from $\pm 1 \mathrm{mJy} \mathrm{b}^{-1}$; the resolution is $45^{\prime \prime} \times 45^{\prime \prime}$, PA $0^{\circ}$. Central panel: $1.4 \mathrm{GHz}$ image (grey scale) of the NVSS pointing containing A697 after new calibration. The $1 \sigma$ level is $0.3 \mathrm{mJy} \mathrm{b}^{-1}$. The resolution is $45^{\prime \prime} \times 45^{\prime \prime}$. Right panel: recalibrated NVSS image (contours) overlaid on the VLA-C array $1.4 \mathrm{GHz}$ image (same as Fig. 4). The first contour is $\pm 0.3 \mathrm{mJy} \mathrm{b}^{-1}$, contours are spaced by a factor of 2. The dashed circle in each panel highlights the portion of the field referred to in Sect. 3.1.

avoid attenuation from the primary beam. We thus obtained a

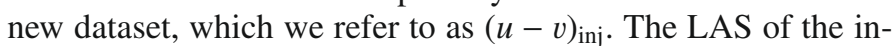
jected fake radio halos (i.e., the diameter of the largest sphere of the model) ranges from $240^{\prime \prime}$ to $330^{\prime \prime}$, corresponding to a linear scale in the range 1 to $1.4 \mathrm{Mpc}$ at the redshift of A 697. The total flux density values injected, $S_{\text {inj, }}$, range from 50 to $70 \mathrm{mJy}$. We then imaged each new dataset $(u-v)_{\text {inj }}$ with the same parameters that we used to produce the final low resolution image of the radio halo (see Fig. 2), and measured the flux density and LAS in each fake radio halo imaged. We note that both the fake and the true radio halos are imaged simultaneously, allowing a direct comparison between the two sources.

In general, we found that the component of the largest angular size (i.e., the one of lowest surface brightness) is partially lost in the imaging process. In contrast, the central highest surface brightness regions are well imaged. These general results are in agreement with what is found in VGD08.

An injected fake halo with $S_{\text {inj }}=60 \mathrm{mJy}$ and LAS $=330^{\prime \prime}$ most closely reproduces the emission observed at the cluster centre, i.e., $S \simeq 48 \mathrm{mJy}$ and LAS $\simeq 300^{\prime \prime}$ (see Sect. 2.3). Based on these tests, we concluded that the expected flux density loss of the radio halo at $325 \mathrm{MHz}$ is at most $\sim 20 \%$. Most of this flux density is lost in the largest and lowest surface brightness component. The LAS of the true halo is $\sim 10 \%$ smaller than that injected.

\subsection{The $610 \mathrm{MHz}$ data and the "revised" spectrum}

Following the same procedure, we estimated the flux density loss at $610 \mathrm{MHz}$. A fake halo with LAS $=330^{\prime \prime}$ (same choice as for the injection at $325 \mathrm{MHz}$ ) and $S_{\text {inj }}=18 \mathrm{mJy}$ most accurately reproduces the radio halo observed at $610 \mathrm{MHz}$. The resulting differential flux density loss between $325 \mathrm{MHz}$ and $610 \mathrm{MHz}$ is $\sim 20 \%$. Based on these results, we estimated that the intrinsic flux density of the radio halo in A 697 is $\sim 57 \mathrm{mJy}$ at $325 \mathrm{MHz}$ and $\sim 20 \mathrm{mJy}$ at $610 \mathrm{MHz}$ (accounting for the $12 \%$ flux density correction derived in Sect. 3.2).

In Fig. 6, we present the integrated spectrum of the halo between 325 and $610 \mathrm{MHz}$ taking into account the losses in flux density (the dashed line connects the two values indicated by the star symbols). The resulting spectral index is $\alpha_{\text {rev }} \sim 1.7$. We conclude that A 697 hosts a very steep spectrum radio halo. The flux density we measured at $1.4 \mathrm{GHz}$ can be considered a lower limit, the upper one being the extrapolation of the "revised" spectrum

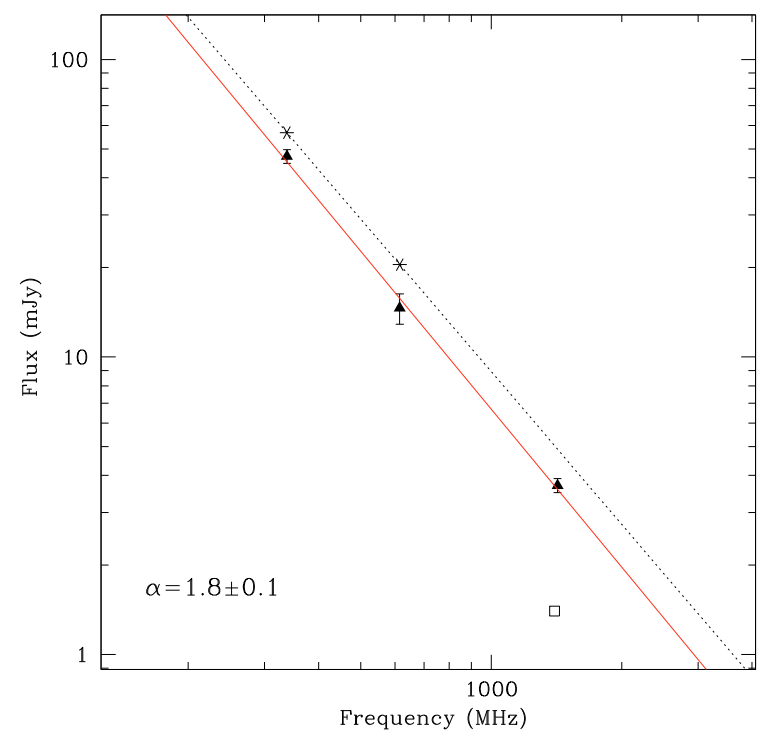

Fig. 6. Integrated radio spectrum of the halo. The red solid line is the linear fit to the data (filled triangles), weighted for uncertainties. The dashed line connects the two values of flux density including the estimated losses at 325 and $610 \mathrm{MHz}$ (stars). The open square is the estimated flux density of the radio halo in the NVSS.

between $325 \mathrm{MHz}$ and $610 \mathrm{MHz}$. This leads to an "expected" flux density value $S_{1.4 \mathrm{GHz}} \simeq 5 \mathrm{mJy}$ over the same region considered in this paper. Future high sensitivity observations at this frequency may allow us to constrain the high frequency end of the radio halo spectrum.

\section{Discussion}

We have presented observational evidence that A 697 hosts a very steep spectrum giant radio halo.

This source has observational properties similar to those of A 521, which we consider the prototypical example of ultrasteep-spectrum radio halos. In particular, it is barely detectable at $1.4 \mathrm{GHz}$ with the current instruments, and with the observed values $\log P_{1.4 \mathrm{GHz}}(\mathrm{W} / \mathrm{Hz})=23.95$ and $L_{\mathrm{X}[0.1-2.4] \mathrm{keV}}=$ $1.06 \times 10^{45} \mathrm{erg} \mathrm{s}^{-1}$, it lies below the well-known $\log P_{1.4 \mathrm{GHz}^{-}}$ $\log L_{\mathrm{X}}$ correlation for giant radio halos (see Brunetti et al. 2009 for a review). The source becomes stronger and considerably more extended at lower frequencies. This is different 
from what is observed for radio halos of spectral index $\alpha \sim$ $1.2-1.4$, whose overall morphology and size do not change appreciably towards lower frequencies (see for instance A 2744 and A 2219, Orrú et al. 2007; A 2319, Feretti et al. 2001; MACS J 0717.5+3745, van Weeren et al. 2009 and Bonafede et al. 2009; RXCJ2003.5-2323; Giacintucci et al. 2009). Based on literature data, Giovannini et al. (2009) reported on four more halos with very steep integrated spectra; however, the different resolutions and $u-v$ coverages, and the lack of accurate subtraction of individual embedded radio sources in the heterogenous datasets, may have affected the shape and spectral index of the integrated spectra.

The observational connection between major cluster mergers and radio halos is a fairly well established result, both on the basis of individual studies (examples of multiband analysis may be found in Govoni et al. 2004; van Weeren et al. 2009; Bonafede et al. 2009; Giacintucci et al. 2009) and on statistical properties of large samples (see for instance Buote 2001 and VGB08). On the other hand, it has been suggested that the majority of radio halos should be generated during more common but less energetic mergers, for example between a massive cluster and a much smaller subcluster (of mass ratio $\gtrsim 5$ ), or between two similar clusters of mass $M \lesssim 10^{15} M_{\odot}$, which would trigger the formation of radio halos observable only up to few hundred $\mathrm{MHz}$ (Cassano et al. 2006 and Cassano 2009). These elusive radio halos have so far been missed by large surveys mainly due to their steep spectrum, which requires very sensitive observations at frequencies $v \leq 1 \mathrm{GHz}$. The discovery of the ultra-steep-spectrum radio halo in A 521 (Brunetti et al. 2008) provides observational support in favour of this idea. The very steep spectrum radio halo found in A 697 offers a chance to test the hypothesis of a connection between "low frequency radio halos" and less energetic cluster mergers.

\subsection{The merger in the cluster A 697}

Optical and X-ray observations are crucial tools for investigating cluster dynamics. A detailed optical analysis of A 697 was carried out in G06, who found that the cluster is significantly far from dynamical relaxation. In particular, the observed complex dynamical state of A 697 might be explained either as an ongoing process of multiple accretion of small $(\lessgtr 0.2 \mathrm{Mpc})$ clumps by a very massive cluster, or as the result of a past merger event. The scenario may be even more complicated (major merger followed by multiple accretion), as suggested by the absence of a cool core in this cluster (Bauer et al. 2005). G06 concluded that it is difficult to obtain more details about the type of merger in A 697, since we are probably viewing the system at a small angle to the line of sight. Analysis of archival Chandra X-ray observations (G06) detected significant substructure in the core region, with three clumps of emission within $\sim 200 \mathrm{kpc}$ from the centre, confirming the cluster complex dynamical state.

We reanalysed the public archive Chandra ACIS-I observation of A 697 (19.8 ks, OBSID 4271, presented also in G06). In Fig. 7, we report our wavelet-reconstructed Chandra image of A 697, with $325 \mathrm{MHz}$ contours of the radio halo superimposed (same image as right panel of Fig. 2). The X-ray image was obtained by applying the wavelet decomposition tool (Vikhlinin et al. 1997) to the cluster image in the 0.5-9 keV energy band, divided for the exposure map and background subtracted (see Giacintucci 2007, and references therein).

As is clear from Fig. 7, the overall X-ray morphology of the cluster is elliptical, and elongated along the north-north west/south-south east (NNW/SSE) direction. The extent and

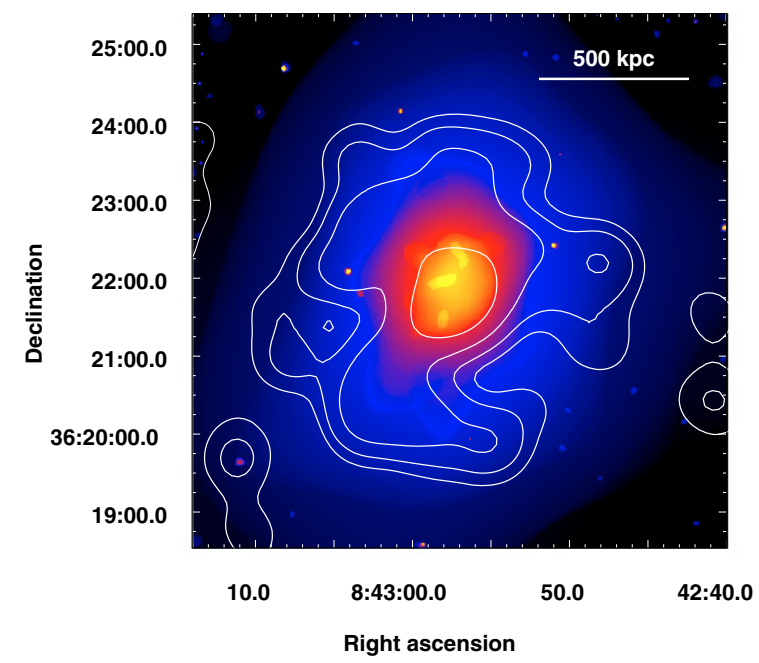

Fig. 7. Wavelet-reconstructed Chandra image of A697, in the $0.5-9 \mathrm{keV}$ band. The image is corrected for the exposure and background subtracted. Overlaid are the $325 \mathrm{MHz}$ contours of the radio halo. The radio image is the same as right panel of Fig. 2.

shape of the radio emission are in agreement with both the size of the X-ray emitting region and the slight elongation along the $\mathrm{NNW/SSE}$ axis. The peak of the radio emission also covers the inner $\sim 200 \mathrm{kpc}$ of the core region, where the three X-ray subclumps are located. The cluster temperature and hardness ratio distributions were derived in Giacintucci (2007), with the calibration files available at the time. Gradients between the inner $\sim 200 \mathrm{kpc}$ core region and the surrounding areas were found in both the temperature and hardness ratio, in agreement with the proposed overall picture that A 697 is dynamically unrelaxed. Our analysis favours the multiple merger scenario, but unfortunately the short exposure time of the Chandra observations does not allow a more quantitative analysis.

\subsection{Origin of the radio halo}

Theoretically, cosmic ray protons are expected to be the dominant non-thermal particle component in the ICM, due to their long lifetime (see Blasi et al. 2007, for a recent review). However, current observations in different bands constrain the energy density of the non-thermal component to be $<10 \%$ of the thermal gas in the central Mpc region of galaxy clusters (e.g. Brunetti et al. 2007; Churazov et al. 2008; Aharonian et al. 2009a,b; Laganá et al. 2010).

As mentioned in the introduction, the two main physical processes producing the extended diffuse synchrotron emission in giant radio halos are probably the injection of secondary electrons by means of proton-proton collisions (hadronic models, e.g. Dennison 1980; Blasi \& Colafrancesco 1999; Pfrommer \& Ensslin 2004), and the in situ reacceleration of relativistic electrons by MHD turbulence generated in the ICM during clustercluster mergers (reacceleration models, Brunetti et al. 2001, 2004; Petrosian 2001; Fujita et al. 2003; Cassano \& Brunetti 2005).

Radio halos with very steep spectrum, i.e., $\alpha \geq 1.6$, are suitable targets for constraining these models and are more easily explained by a turbulent re-acceleration scenario (Brunetti et al. 2008). In the context of the hadronic scenario, clusters hosting these radio halos must contain a very large population of cosmic ray protons. The observations presented in this paper suggest 
that Abell 697 hosts a very steep spectrum giant halo and this allows a prompt test of the hadronic model.

We assume that the giant radio halo in A 697 is of hadronic origin and discuss the consequences of this hypothesis on the physical properties of the ICM. The decay chain that we consider for the injection of secondary particles in the ICM produced by $\mathrm{p}-\mathrm{p}$ collisions is (Blasi \& Colafrancesco 1999)

$$
\begin{array}{r}
\mathrm{p}+\mathrm{p} \rightarrow \pi^{0}+\pi^{+}+\pi^{-}+\ldots \\
\pi^{0} \rightarrow \gamma \gamma \\
\pi^{ \pm} \rightarrow \mu+v_{\mu} \quad \mu^{ \pm} \rightarrow \mathrm{e}^{ \pm} v_{\mu} v_{\mathrm{e}}
\end{array}
$$

which is a threshold reaction that requires protons of kinetic energy higher than $T_{\mathrm{p}} \approx 300 \mathrm{MeV}$.

Based on the assumption that secondary electrons are not accelerated by other mechanisms, their spectrum approaches a stationary distribution due to the competition between injection and energy losses (Dolag \& Ensslin 2000)

$N_{\mathrm{e}}^{ \pm}(p)=\frac{1}{\left|\left(\frac{\mathrm{d} p}{\mathrm{~d} t}\right)_{\text {loss }}\right|} \int_{p}^{p_{\max }} Q_{\mathrm{e}}^{ \pm}(p) \mathrm{d} p$,

where $Q_{\mathrm{e}}^{ \pm}$is the injection rate of secondary electrons and radiative losses, which dominate for $\gamma>10^{3}$ electrons in the ICM, are given by (Sarazin 1999)

$$
\left|\left(\frac{\mathrm{d} p}{\mathrm{~d} t}\right)_{\mathrm{loss}}\right| \simeq 3.3 \times 10^{-32}\left(\frac{p / m_{\mathrm{e}} c}{300}\right)^{2}\left[\left(\frac{B_{\mu \mathrm{G}}}{3.2}\right)^{2}+(1+z)^{4}\right] .
$$

We follow standard formulae to calculate the injection rate of secondary electrons (Moskalenko \& Strong 1998; Blasi \& Colafrancesco 1999; Brunetti \& Blasi 2005) and use Dermer's fitting formulae for the inclusive $\mathrm{p}-\mathrm{p}$ cross section (Dermer 1986), which allow us to describe separately the rates of generation of $\pi^{-}, \pi^{+}$, and $\pi^{o}$.

We assume a power law distribution of relativistic protons, $N_{\mathrm{p}}(p)=K_{\mathrm{p}} p^{-s}$, in which case the spectrum of secondaries at high energies, $\gamma>10^{3}$, is $N_{\mathrm{e}}(p) \propto p^{-(s+1)} \mathcal{F}(p)$, where $\mathcal{F}$ accounts for the log-scaling of the $\mathrm{p}-\mathrm{p}$ cross section at high energies and makes the spectral shape slightly flatter than $p^{-(s+1)}$ (Brunetti \& Blasi 2005; Brunetti 2009).

The synchrotron emissivity from secondary $\mathrm{e}^{ \pm}$is also obtained via the standard formulae (Rybicki \& Lightman 1979; see also Dolag \& Ensslin 2000)

$$
\begin{aligned}
J_{\text {syn }}(v) & =\sqrt{3} \frac{e^{3}}{m_{\mathrm{e}} c^{2}} B \int_{0}^{\pi / 2} \mathrm{~d} \theta \sin ^{2} \theta \int \mathrm{d} p N_{\mathrm{e}}(p) F\left(\frac{v}{v_{\mathrm{c}}}\right) \\
& \propto n_{\mathrm{th}} \epsilon_{\mathrm{CR}} \frac{B^{1+\alpha}}{B^{2}+B_{\mathrm{cmb}}^{2}} v^{-\alpha},
\end{aligned}
$$

where

$\epsilon_{\mathrm{CR}}=K_{\mathrm{p}} \int p^{-s} T_{\mathrm{p}} \mathrm{d} p$

is the energy density of cosmic ray protons $\left(T_{\mathrm{p}}\right.$ is the kinetic energy of protons), $F$ is the synchrotron Kernel (Rybicki \& Lightman 1979), $v_{\mathrm{c}}$ is the critical frequency, and $\alpha \simeq s / 2-\Delta$, $\Delta \sim 0.1-0.15$ due to the logarithmic scaling of the cross-section (e.g., Brunetti 2009). We adopt a value of the spectral index of the halo $\alpha=1.75$, which is consistent with our observational findings and implies a spectral slope of the cosmic ray protons $s=3.8$.

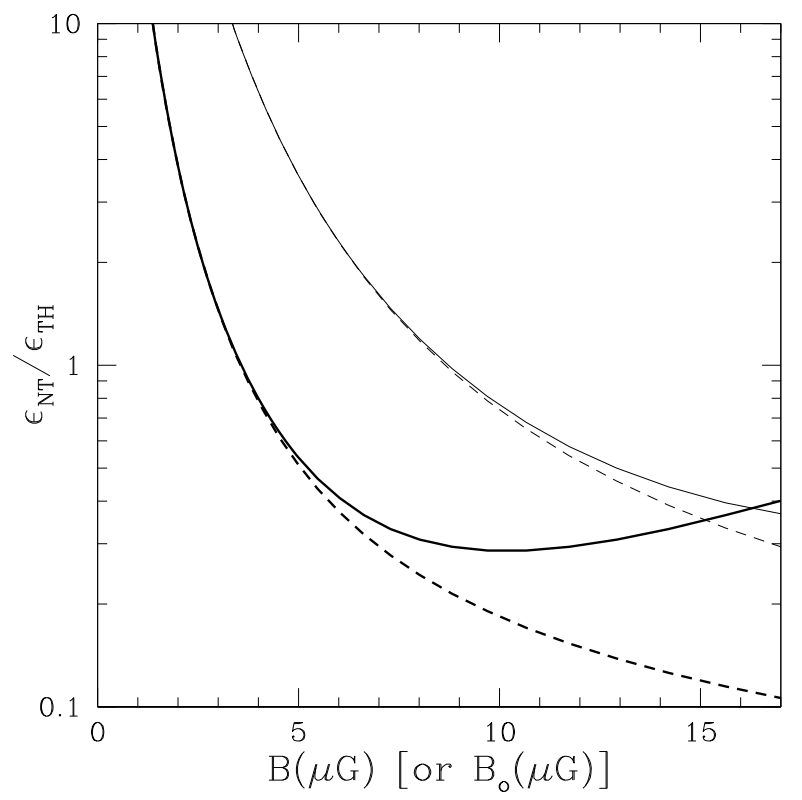

Fig. 8. The ratios of the energy densities of relativistic protons to those of the thermal ICM (dashed lines) and of the non-thermal (relativistic protons and magnetic field) and to the thermal ICM (solid lines) are shown as a function of magnetic field. All energy densities are calculated within a distance $r \sim 3 r_{\mathrm{c}}$, i.e., roughly the region filled by the radio halo. Thick lines refer to Model 1 , in which case $B$ is that within $2.5 r_{\mathrm{c}}$. Thin lines are obtained by assuming that the magnetic field energy-density scales with the thermal one, $B=B_{0}\left(n_{\mathrm{th}} / n_{\mathrm{o}}\right)^{1 / 2}$ (e.g. Govoni \& Feretti 2004; with thermal quantities taken from Bonamente et al. 2006), and by leaving the radial profile of relativistic protons free to vary with distance (up to $r \sim 2.5 r_{\mathrm{c}}$ while at larger distances it is assumed to scale with thermal one as in Model 1) to match the observed synchrotron profile; in this latter case, the $x$-axis shows the central field value, $B_{0}$.

The diffuse emission of the radio halo is centered on the cluster core region and grossly resembles the X-ray morphology both in terms of extension and elongation (Fig. 7). The radio profile at $325 \mathrm{MHz}$, however, is flatter than the X-ray profile, and drops by only a factor 5-6 over a distance range of 2.5-3 core radii $(\sim 500 \mathrm{kpc})$, suggesting a broad spatial distribution of the relativistic particles. Thus, we used a $\beta$-model for the spatial distribution of the cluster thermal gas (with parameters taken from Bonamente et al. 2006: $\beta=0.6, n_{o}=9.8 \times 10^{-3} \mathrm{~cm}^{-3}, r_{\mathrm{c}}=42^{\prime \prime}$, i.e., $\approx 180 \mathrm{kpc}$, and gas temperature $k T=10 \mathrm{keV}$ ) and adopt a model (Model 1) of the distribution of relativistic components in Abell 697 where the energy density of both cosmic ray protons and magnetic field are constant with radius out to a distance $=2.5 r_{\mathrm{c}}$, and scale with that of the thermal gas at larger distances. This model gives a very good representation of the observed halo radial profile out to a distance of about $2.5 r_{\mathrm{c}}$ and, within the uncertainties, it is also in line with the observed profile at large distance (regardless of the strength of the magnetic field at $\leq 2.5 r_{\mathrm{c}}$ distance).

In Fig. 8, we show the ratio of the energy density of nonthermal (relativistic protons and magnetic field) and thermal components in Abell 697 corresponding to a hadronic origin of the radio halo. For $B \leq 5 \mu \mathrm{G}$, we find that relativistic protons are required to store an energy comparable to (or higher than) that of the thermal ICM. The non-thermal energy content reaches a minimum for $B \approx 10 \mu \mathrm{G}, \epsilon_{\mathrm{NT}} \approx 1 / 3 \epsilon_{\mathrm{TH}}$, implying an important dynamical contribution of the non-thermal components in the cluster. In addition, we point out that Model 1 provides only 
a lower limit to the energy of the non-thermal components for two main reasons:

- As soon as we also include the contribution from the tail of the proton energy distribution at sub-relativistic energies $(E<1 \mathrm{GeV})$, the required energy budget is much higher than that in Fig. 8 due to the steep proton spectrum, $\epsilon_{\mathrm{CR}} \propto p_{\min }^{-s+3}$.

- The rather unphysical assumption in Model 1 that the energy density of the non-thermal components is constant with radius (up $2.5 r_{\mathrm{c}}$ ) yields a lower limit to the energy density of the cosmic ray protons required by the hadronic scenario. As soon as the magnetic field strength in Abell 697 is allowed to decrease with distance from the cluster centre, the energy density of relativistic protons must increase with radius and their total energy budget increases (Fig. 8).

We conclude that the very large energy budget necessary for the non-thermal components if the halo is of a hadronic origin tends to exclude this scenario.

\section{Summary and conclusions}

We have presented 325 GMRT high sensitivity observations of the giant radio halo in A 697, and performed an accurate study of its integrated spectrum.

The largest extent of the radio halo at $325 \mathrm{MHz}$ is $\sim 5^{\prime}$ (corresponding to $1.3 \mathrm{Mpc} \mathrm{h}_{70}^{-1}$ ), which is considerably larger than at higher frequencies. The radio spectrum, determined with three data points in the frequency range $325 \mathrm{MHz}-1.4 \mathrm{GHz}$, has a spectral index $\alpha_{325 \mathrm{MHz}}^{1.4 \mathrm{GHz}}=1.8 \pm 0.1$. An accurate analysis of the fraction of flux density that might be missed in our observations beacuse of the limited the $u-v$ coverage at short spacings, inferred a spectral index $\alpha_{\text {rev }}=1.7$. We thus conclude that A 697 hosts a very steep spectrum radio halo.

A qualitative analysis of the X-ray emission from the ICM suggests that this cluster is unrelaxed. A comparison with the $325 \mathrm{MHz}$ emission from the radio halo shows that the non-thermal and thermal emissions have similar morphologies. Though at a qualitative level, the observations are in agreement with the idea that A 697 is the result of a multiple merger.

As in the case of A 521, we have demonstrated that the very steep spectrum of the halo appears to exclude a hadronic origin, which would require an unplausibly high energy budget. On the other hand, models that produce radio halos by means of turbulent acceleration predict that a large number of halos in the Universe have a very steep spectrum. This will be tested as soon as LOFAR and LWA become operational.

Acknowledgements. We thank the staff of the GMRT for their help during the observations. GMRT is run by the National Centre for Radio Astrophysics of the Tata Institute of Fundamental Research. This research is partially funded by INAF and ASI through grants PRIN-INAF 2007, PRIN-INAF 2008 and ASIINAF I/088/06/0. This research has made use of the NASA/IPAC Extragalactic Database (NED) which is operated by the Jet Propulsion Laboratory, California Institute of Technology, under contract with the National Aeronautics and Space Administration.

\section{References}

Abell, G. O., Corwin, H. G. Jr., \& Olowin, R. P. 1989, ApJS, 70, 1 Aharonian, F., Akhperjanian, A. G., Anton, G., et al. 2009a, A\&A, 495, 27 Aharonian, F., Akhperjanian, A. G., Anton, G., et al. 2009b, A\&A, 502, 437 Bauer, F. E., Fabian, A. C., Sanders, J. S., et al. 2005, MNRAS, 359, 1481 Bacchi, M., Feretti, L., Giovannini, G., et al. 2003, A\&A, 400, 465 Blasi, P., \& Colafrancesco, S. 1999, APh, 12, 169

Blasi, P., Gabici, S., \& Brunetti, G. 2007, Int. J. Mod. Phys. A, 22, 681 Bonafede, A., Feretti, L., Giovannini, G., et al. 2009, A\&A, 503, 707 Bonamente, M., Joy, M. K., LaRoque, S. J., et al. 2006, ApJ, 647, 25 Brunetti, G. 2004, JKAS, 37, 493

Brunetti, G. 2009, Rev. Mex. Astron. Astrophys., 36, 201

Brunetti, G. 2009, A\&A, 508, 599

Brunetti, G., \& Blasi, P. 2005, MNRAS, 363, 1173

Brunetti, G., Setti, G., Feretti, L., et al. 2001, MNRAS, 320, 365

Brunetti, G., Venturi, T., Dallacasa, D., et al. 2007, ApJ, 670, L5

Brunetti, G., Giacintucci, S., Cassano, R., et al. 2008, Nature, 455, 944

Brunetti, G., Cassano, R., Dolag, K., et al. 2009, A\&A, 507, 661

Buote, D. A. 2001, ApJ, 553, L15

Cassano, R. 2009, in The Low Frequency Radio Universe, ed. D. J. Saikia, D. A.

Green, Y. Gupta, \& T. Venturi, ASP Conf. Ser., 407, 223

Cassano, R., \& Brunetti, G. 2005, MNRAS, 357, 1313

Cassano, R., Brunetti, G., \& Setti, G. 2006, MNRAS, 369, 1577

Churazov, E., Forman, W., \& Vikhlinin, A. 2008, MNRAS, 388, 1062

Condon, J. J., Cotton, W. D., Greisen, et al. 1998, AJ, 115, 1693

Dahle, H., Kaiser, N., Irgens, R. J., et al. 2002, ApJS, 139, 313

Dallacasa, D., Brunetti, G., Giacintucci, S., et al. 2009, ApJ, 699, 1288

De Filippis, E., Sereno, M., Bautz, M. W., et al. 2005, ApJ, 625, 108

Dennison, B. 1980, ApJ, 239, L93

Dermer, C. D. 1986, A\&A, 157, 223

Dolag, K., \& Ensslin, T. A. 2000, A\&A, 362, 151

Ebeling, H., Edge, A. C., Bohringer, H., et al. 1998, MNRAS, 301, 881

Feretti, L., Fusco-Femiano, R., Giovannini, G., et al. 2001, A\&A, 373, 106

Ferrari, C., Govoni, F., Schindler, S., et al. 2008, Space Sci. Rev., 134, 93

Fujita, Y., Takizawa, M., \& Sarazin, C. L. 2003, ApJ, 584, 190

Giacintucci, S., PhD Thesis Multiwavelength study of cluster mergers and consequences for the radio emission properties of galaxy clusters, University of Bologna, http: //amsdottorato.cib.unibo.it/353/

Giacintucci, S., Venturi, T., Brunetti, G., et al. 2005, A\&A, 440, 867

Giacintucci, S., Venturi, T., Brunetti, G., et al. 2009, A\&A, 505, 45

Giovannini, G., Tordi, M., \& Feretti, L. 1999, New Astron., 4, 141

Giovannini, G., Bonafede, A., Feretti, L., et al. 2009, A\&A, 507, 1257

Girardi, M., Boschin, W., \& Barrena, R. 2006, A\&A, 455, 45 (G06)

Govoni, F., \& Feretti, G. 2004, Int. J. Mod. Phys. D., V. 13, 8, 1549

Govoni, F., Feretti, L., Giovannini, G., et al. 2001, A\&A, 376, 803

Govoni, F., Markevitch, M., Vikhlinin, A., et al. 2004, ApJ, 605, 695

Jaffe, W. J. 1977, ApJ, 212, 1

Kempner, J. C., \& Sarazin, C. L. 2001, ApJ, 548, 639

Laganá, T. F., de Souza, R. S., \& Keller, G. R. 2010, A\&A, 510, 76

Metzger, M. R., \& Ma, C. P. 2000, AJ, 120, 2879

Moskalenko, I. V., \& Strong, A. W. 1998, ApJ, 493, 694

Orrú, E., Murgia, M., Feretti, L., et al. 2007, A\&A, 467, 943

Ota, N., \& Mitsuda, K. 2004, A\&A, 428, 757

Petrosian, V. 2001, ApJ, 557, 560

Pfrommer, C., \& Enßlin, T. A. 2004, JKAS, 37, 455

Rengelink, R. B., Tang, Y., de Bruyn, A. G., et al. 1997, A\&AS, 124, 259

Rybichi, G. B., \& Lightman, A. P. 1979, Astron. Q., 3, 199, 1980

Sarazin, C. L. 1999, ApJ, 520, 529

van Weeren, R. J., Rottgering, H. J. A., Brüggen, M., et al. 2009, A\&A, 505, 991 Venturi, T., Giacintucci, S., Brunetti, G., et al. 2007, A\&A, 463, 937 (VGB07)

Venturi, T., Giacintucci, S., Dallacasa, D., et al. 2008, A\&A, 484, 327 (VGD08)

Venturi, T., Giacintucci, S., Cassano, R., et al. 2009, in The low frequency Universe, ed. D. J. Saikia, D. A. Green, Y. Gupta, \& T. Venturi, ASPC 407, 232

Vikhlinin, A., Forman, W., \& Jones, C. 1997, ApJ, 474, L7 